\title{
Quantum reflection of rare-gas atoms and clusters from a grating
}

\author{
G. Rojas-Lorenzo* and J. Rubayo-Soneira ${ }^{\dagger}$ \\ Instituto Superior de Tecnologías y Ciencias Aplicadas (InsTEC), Universidad de La Habana, Avenida Salvador Allende No. 1110, \\ Quinta de Los Molinos, La Habana 10400, Cuba \\ S. Miret-Artés ${ }^{\ddagger}$ \\ Instituto de Física Fundamental, Consejo Superior de Investigaciones Científicas, Serrano 123, 28006 Madrid, Spain \\ E. Pollak ${ }^{\S}$ \\ Department of Chemical and Biological Physics, Weizmann Institute of Science, 76100, Rehovot, Israel
}

(Received 19 July 2018; published 4 December 2018)

\begin{abstract}
Quantum reflection is a universal property of atoms and molecules when scattered from surfaces in ultracold collisions. Recent experimental work has documented the quantum reflection and diffraction of He atoms, dimers, trimers, and neon atoms when reflected from a grating. Conditions for the observation of emerging beam resonances have been discussed and measured. In this paper, we provide a theoretical simulation of the quantum reflection from a grating for those systems. We confirm the universal dependence on the incident de Broglie wavelength with the threshold angles where the emerging beam resonances are observed. However, the angular dependence of the reflection efficiencies, that is, the ratio of scattered intensity into specific diffraction channels relative to the total intensity is found to be dependent on the details of the particle surface interaction.
\end{abstract}

DOI: 10.1103/PhysRevA.98.063604

\section{INTRODUCTION}

Optical effects (such as refraction, diffraction, and interferometry) observed in matter waves instead of light are determined by the interaction between the corresponding particles and measuring devices. The particle-specific interaction leads to some distortion and reduction of their visibility. The so-called Talbot effect [1] or near-field interference effect is a good illustration of the differences between optical and matter wave effects. Quantum carpets are observed in the near field when gratings are illuminated by coherent light and particle beams. However, the patterns observed when using particles is distorted as compared to the same effect when using photons due to the particle grating specific interaction (Talbot-Beeby [2]) which is typified by a well in the interaction potential close to the surface of the grating. When heavy particles such as big molecules and clusters are used, diffraction patterns are governed mainly by van der Waals interactions and a strong reduction of the fringe visibility is observed [3].

It is thus of interest to reduce such matter-wave-related distortions as much as possible. Recently, Zhao et al. [4] proposed and demonstrated that this is possible thanks to the well-known effect of quantum reflection which is a key effect in many cold and ultracold gas-phase collisions as well as the scattering of particles by solid surfaces. Lennard-Jones

\footnotetext{
*german@instec.cu

$\dagger$ jrs@instec.cu

${ }^{\ddagger}$ s.miret@iff.csic.es

§eli.pollak@weizmann.ac.il
}

and Devonshire [5] first recognized this behavior in the atomsurface context and Kohn [6] showed later on that quantum reflection leads to a zero sticking probability at threshold, pointing out that this effect is a clear quantum interference effect between the incoming and reflected waves.

It is well understood that at threshold the maximum of the scattered wave function is observed far away from the grating, due to the very long de Broglie wavelength of the particle at the low energies involved. This has led to the notion that quantum reflection takes place far away from the grating or surface, with a distance of typically tens or hundreds of nanometers where the surface-induced forces are too weak to dissociate fragile bonds such as in $\mathrm{He}_{2}$ [7] and one cannot consider a classical turning point for such reflection, which would immediately dissociate a weakly bound molecule such as the He dimer [8,9]. This large distance then presumably weakens the distortions in the diffraction patterns due to the short-range interactions with the surface. In this context, Zhao et al. [4], in their experimental study of the scattering of $\mathrm{He}, \mathrm{He}_{2}$, and $\mathrm{D}_{2}$ by an echelette or blazed ruled grating, observed a "universal" dependence of the so-called emerging beam resonances (or threshold resonances in atom-surface scattering [10]) which occur when a diffraction channel just becomes open or closed. The "universality" expresses itself in the fact that the threshold depends only on the de Broglie wavelength of the incident beam but not on the characteristics of the particle-surface interaction. The threshold incident angles are called Rayleigh angles. In this same study it was claimed that not only the threshold was universal but also the incident angle dependence of the "efficiency" (the ratio of the diffraction peak to the total intensity) is universal. 
As discussed more recently [11,12], the fact that the maximal density in the scattered wave function appears far away from the surface does not mean that the details of the quantum reflection really are universal and independent of the moiety observed. Quantum reflection is mainly governed by the long-range attractive van der Waals (vdW)-Casimir potential tail which falls off faster than $r^{-2}$. [13]. Senn [14] showed that for general one-dimensional potentials which vanish as the coordinate goes to $\pm \infty$, the reflection probability goes to unity at threshold conditions except when the potential supports a zero-energy resonance state. The reflection coefficient decreases from unity as the incident kinetic energy increases according to $|R| \sim 1-2 k b \sim \exp (-2 k b)$, where $k$ is the incident wave vector and $b$ a characteristic length which depends on the specifics of the particle-grating interaction. This universal behavior is a direct result of boundary conditions and continuity of the wave function and its derivative $[12,14]$.

In the semiclassical framework, however, the analysis of quantum reflection has concentrated on the fact that in the regime of linear dependence on $k$ there is a failure of the semiclassical description of the scattering dynamics [15]. Far away from the grating or surface the long-range attractive potential exhibits a region in which the local de Broglie wavelength is not slowly varying, invalidating a semiclassical description in this so-called badlands region of the interaction potential. Quantum reflection was thus associated with this badlands region where "quantality" is high [16]. For $\mathrm{He}$ atoms, the badlands region of the potential is typically located at distances of several hundreds of atomic units from the grating or surface and thus would depend only on the longrange part of the interaction potential.

Recently, we have shown theoretically [11] that the quantum reflection of $\mathrm{He}$ atoms from a grating is determined not only by the long-range interaction potential but also by its short-range properties. We emphasized that this short-range region is critical for obtaining theoretical reflection probabilities and diffraction patterns which are in fairly good agreement with the experimental results. These calculations were carried out by using the close-coupling (CC) formalism [17], which is numerically exact when convergence is reached. To distinguish between quantum reflection and "classical" reflection due to the turning point of the repulsive part of the interaction, absorbing boundary conditions which prevented the classical reflection from occurring were employed. In Ref. [12] we also showed that the badlands region of the interaction potential is immaterial since the wavelength of the scattering particles at threshold is much longer than the spatial extension of the badlands region. In other words, this region does not provide a qualitative guide to the occurrence of quantum threshold reflection.

Quantum reflection thus presents theory with a number of challenges. One is to show that measured quantum reflection probabilities may be simulated by theory using the wellknown long-range interaction potential but also considering the periodicity of the grating. A second challenge is to display the universality of the Rayleigh angles and their dependence on the incident wavelength only. A third question is the extent of this universality, does it also include the dependence of the efficiency on the incident scattering angle?
The purpose of this present work is to answer these challenges. To set the stage we show that with reasonable interaction potentials it is possible to simulate rather well the quantum reflection of $\mathrm{He}, \mathrm{He}_{2}, \mathrm{He}_{3}$, and $\mathrm{Ne}$ on a "standard" or regular grating. Comparison with experiment where possible is good, the quantum reflection of $\mathrm{He}_{2}$ has only been measured for a blazed grating so the theoretical results for $\mathrm{He}_{2}$ have yet to be validated experimentally. As expected, for all systems considered we observe the universality of the Rayleigh angles in their dependence on the incident wavelength only. However, the dependence of the efficiency on the incident angle is not universal and does depend on the specifics of the interaction. Although we are considering here only a "standard" grating rather than the ruled grating used in the experiments [4] this specificity is general and a consequence of the theory of quantum reflection and its dependence on energy in the linear regime. In Sec. II we review the theory needed to implement the computations, in Sec. III we present the results. The paper ends with a discussion of the various aspects of the quantum reflection phenomenon.

\section{THEORY}

The experiment we want to analyze has been described in detail in Ref. [18]. The reflection grating is assumed to be in the $x$ direction and consists of a 56-mm-long microstructured array of 110-nm-thick, $10-\mu \mathrm{m}$-wide, and 5-mm-long parallel chromium strips on a flat quartz substrate. The period of the strips $d$ is $20 \mu \mathrm{m}$. With this geometry, the quartz surface between the strips is completely shadowed by them for all the incidence angles used. Quantum reflection probabilities as well as diffraction patterns were measured at different source temperatures $T_{0}$ (ranging typically from 8 to $40 \mathrm{~K}$ ) and pressures around $P_{0}=6-8$ bar. In the cryogenic free jet expansion of incident particles, the kinetic energy is given by $E_{i}=(5 / 2) k_{B} T_{0}$ where $k_{B}$ is the Boltzmann constant [19]. The incident grazing angle $\theta_{i}$ is usually varied between 0.4 and $15 \mathrm{mrad}$ and measured with respect to the grating surface plane. The diffraction angles $\theta_{n}$ are given by the conservation of the momentum or Bragg's law

$$
\cos \theta_{i}-\cos \theta_{n}=\frac{n \lambda}{d},
$$

where $\lambda$ is the de Broglie wavelength of the incident particle and the diffraction order is given by $n$. Negative diffraction orders correspond to diffraction angles close to the surface grating; that is, energy in the perpendicular direction is transferred to the parallel direction. Final results are very often plotted as a function of the corresponding perpendicular wave vector along the $z$ direction

$$
k_{\text {perp }} \simeq \frac{\sqrt{5 m k_{B} T_{0}}}{\hbar} \sin \theta_{i},
$$

where $m$ is the atomic mass of the incident particle. When considering clusters such as $\mathrm{He}_{2}$ and $\mathrm{He}_{3}$, this expression is rewritten as

$$
k_{\text {perp }} \simeq N \frac{\sqrt{5 m k_{B} T_{0}}}{\hbar} \sin \theta_{i},
$$

with $N=2,3$, respectively [20]. 
As previously used [11], the two-dimensional model potential between the incoming particles and the grating is assumed to be a product of two functions

$$
U(x, z)=V(z) h(x),
$$

where $V(z)$ describes the interaction along the perpendicular coordinate $z$ and $h(x)$ the periodic grating along the horizontal coordinate $x$. In all our computations we do not take into consideration the internal motion of the approaching molecule which is considered to be structureless. The first factor is taken to be a Morse potential $V_{M}(z)$, at short distances, and an attractive van der Waals-Casimir tail $V_{C}$, at large distances,

$$
V(z)= \begin{cases}V_{M}(z)=D\left[e^{-2 \chi z}-2 e^{-\chi z}\right], & z<\bar{z}, \\ V_{C}(z)=-\frac{C_{4}}{(l+z) z^{3}}, & z \geqslant \bar{z},\end{cases}
$$

where $C_{4}=C_{3} l, C_{3}$ being the vdW coefficient and $l$ a characteristic length which determines the transition from the $\mathrm{vdW}$ $(z \ll l)$ to the Casimir $(z \gg l)$ regime. The matching point $\bar{z}$ is determined by imposing the continuity condition for the interaction potential $\left[V_{M}(\bar{z})=V_{C}(\bar{z})\right]$ and its first derivative $\left[V_{M}^{\prime}(\bar{z})=V_{C}^{\prime}(\bar{z})\right]$. The range of variation of the $C_{3}$ parameter is usually known so that the only real free parameter of the Morse potential in this model is the stiffness parameter $\chi(D$ is determined from the matching point $\bar{z}$ ).

The periodic function describing the grating is written as

$$
h(x)=\sum_{n=-\infty}^{+\infty} \prod\left(\frac{x-n d}{a}\right),
$$

where $a$ is the width of the strips and $d$ the period with $a<d$. The $\prod(y)$ function is the so-called unit impulse function: 0 for $|y|>1 / 2,1$ for $|y|<1 / 2$, and $1 / 2$ for $|y|=1 / 2$. In terms of a Fourier series, $h(x)$ is expressed as

$$
h(x)=\sum_{n=-\infty}^{+\infty} c_{n} e^{i 2 \pi n x / d}
$$

with $c_{0}=a / d, c_{-n}=c_{n}$, and $c_{n}=(a / d) \operatorname{sinc}(n a / d)$, and $\operatorname{sinc}(x)=\sin (\pi x) / \pi x$. When $d=2 a$ (as in the experimental grating of Ref. [18]), the terms beyond the sixth order are in practice no longer significant. The periodic interaction potential can then be expressed as

$$
U(x, z)=\sum_{n=-\infty}^{+\infty} V_{n}(z) e^{i \frac{2 \pi n x}{d}},
$$

where the first term $(n=0)$ is the interaction potential $V_{0}(z)=V(z)$ [see Eq. (5)] and the coupling terms $(n \neq 0)$ are given by

$$
V_{n}(z)=2 \operatorname{sinc}\left(n \frac{a}{d}\right) V(z) .
$$

As has been recently shown [11], the elastic scattering of the incident particles with the grating is theoretically well described by the CC formalism which accounts for the quantum reflection probabilities as well as diffraction patterns. The $\mathrm{CC}$ equations are

$$
\left[\frac{\hbar^{2}}{2 m} \frac{d^{2}}{d z^{2}}+\frac{\hbar^{2}}{2 m} k_{n, z}^{2}-V_{0}(z)\right] \psi_{n}(z)=\sum_{n \neq n^{\prime}} V_{n-n^{\prime}}(z) \psi_{n^{\prime}}(z),
$$

with $\frac{\hbar^{2}}{2 m} k_{n, z}^{2}$ being the $z$ component of the kinetic energy of the scattered particles. The square $z$ component of the wave vector is written as a kinematic relation according to

$$
k_{n, z}^{2}=k_{i}^{2}-\left(k_{i} \sin \theta_{i}+\frac{2 \pi n}{d}\right)^{2},
$$

with $\theta_{i}$ measured with respect to the normal to the surface. Thus, when comparing with experimental results, theoretical positive $n$ diffraction orders correspond to experimental negative ones. For every $n$, the effective potential $V_{0}(z)+$ $\frac{\hbar^{2}}{2 m}\left(k_{i} \sin \theta_{i}+2 \pi n / d\right)^{2}$ in Eq. (10) represents a diffraction channel, whose asymptotic energy is given by the second term. This energy depends on $n$ and the incident scattering conditions (incident energy and polar angle). Open (closed) diffraction channels have a positive (negative) normal kinetic energy $\hbar^{2} k_{n, z}^{2} /(2 m)$. The coupling between channels $V_{n-n^{\prime}}(z)$ is given by Eq. (9) since $n-n^{\prime}$ is always an integer number. The diffraction probabilities are obtained by solving the CC equations [Eq. (10)] with the corresponding boundary conditions [17].

The diffraction intensities or probabilities, obtained by solving the CC equations given by Eq. (10) with the usual boundary conditions [11], are expressed as

$$
I_{n}=\left|S_{n 0}\right|^{2}
$$

where $S_{n n^{\prime}}$ are the elements of the scattering matrix, which give the amplitude of probability for an incident wave at the specular channel $\left(n^{\prime}=0\right)$ and exiting by any of the open diffraction channels labeled by $n$. By construction, the $S$ matrix is unitary. It should be stressed, contrary to some claims in the literature [21], that one should not ignore the closed channels in the computation. Although they are not important in the asymptotic region where the coupling to them vanishes, they do affect strongly the diffraction probabilities as well as the total reflection probability. This is but another indication that quantum reflection is determined by the global potential and not only by the asymptotic form. This implies that numerical convergence needs to be verified with respect to not only the grid size and integration step but also the number of closed channels.

In diffracting systems, when a diffraction channel becomes just open or closed, an emerging or evanescent beam is observed, respectively. Due to the unitarity condition of the $S$ matrix, the diffraction intensities undergo, in general, some abrupt variations. The corresponding kinematic condition (11) fulfills $k_{n, z}^{2}=0$. For a given incident energy and $n$ value, the corresponding incident angle $\theta_{i}$ is called the $n$ th-order Rayleigh angle and this abrupt variation of the intensity is called emerging beam resonance or Rayleigh-Wood anomaly [22] in grating scattering (or threshold resonance in atom-surface scattering [10,23]).

The interaction potential given by Eq. (5) displays classical turning points due to the repulsive part of the Morse potential. To distinguish between quantum reflection and the "normal" reflection from the inner repulsive part of the Morse potential, we impose absorbing boundary conditions [24,25] in the inner part. For this purpose, a Woods-Saxon (WS) potential is introduced as an imaginary part of the diffraction channel 
TABLE I. Parameters of the interaction potential $V(z)$ for the incident particles $\mathrm{He}, \mathrm{He}_{2}, \mathrm{He}_{3}$, and $\mathrm{Ne}$. The stiffness parameter of the Morse potential, $\chi$, is a free parameter fitted to reproduce the corresponding experimental results and $D$ is the well depth. The characteristic lengths $l$ and parameters $C_{3}$ are based on values reported in previous works [4].

\begin{tabular}{lcccc}
\hline \hline Parameters & $\mathrm{He}$ & $\mathrm{He}_{2}$ & $\mathrm{He}_{3}$ & $\mathrm{Ne}$ \\
\hline$\chi\left(\AA^{-1}\right)$ & 0.5 & 0.43 & 0.405 & 0.5 \\
$D(\mathrm{meV})$ & 9.8 & 12.28 & 15.3 & 19.8 \\
$l(\AA)$ & 93 & 93 & 93 & 118.4 \\
$C_{3}\left(10^{-50} \mathrm{Jm}^{3}\right)$ & 3.5 & 7.0 & 10.5 & 7.0 \\
\hline \hline
\end{tabular}

potentials

$$
V_{\mathrm{WS}}=\frac{A}{1+e^{\alpha \chi\left(z-z_{i}\right)}},
$$

which is essentially zero in the physically relevant interaction region and turns on sufficiently rapidly but smoothly at the left edge of the numerical grid for the integration to absorb the flux. The free parameters $A$ and $\alpha$ of the WS potential depend on the system under consideration. Due to this numerical procedure, the resulting scattering matrix $\bar{S}$ is no longer unitary. The diffraction intensities are then given by $\bar{I}_{n}=\left|\bar{S}_{n 0}\right|^{2}$ and the total quantum reflection probability is calculated from

$$
P^{\mathrm{QR}}=\sum_{n}\left|\bar{S}_{n 0}\right|^{2}<1
$$

for each initial condition. Due to the absorbing potential the theoretical diffraction efficiencies are defined as the ratio of the diffraction intensity $\bar{I}_{n}$ to the total quantum reflection probability $P^{\mathrm{QR}}$ rather than to the total incident flux.

\section{RESULTS AND DISCUSSION}

As previously discussed [11], numerical convergence when solving the $\mathrm{CC}$ equations is reached in two steps. First, the grid parameters have to be established. For the four incident particles under study, the initial grid point is between $-10 \AA$ (for $\mathrm{He}$ ) and $-20 \AA$ (for $\mathrm{He}_{3}$ ) and the final integration point is between $500 \AA$ (for $\mathrm{He}$ ) and $1000 \AA$ (for $\mathrm{He}_{3}$ ) and $2000 \AA$ (for $\mathrm{Ne}$ ), the number of points of the grid ranging between 10000 and 20000 . In the second step, the maximum number of open and closed diffraction channels are chosen to be 61 (from $n=-30$ to +30 ). Only one free potential parameter must be fitted for each diffractive system to reproduce the experimental reflection probabilities. The different potential parameters are listed in Table I for each incident particle.

The $C_{3}$ and $l$ values are adapted from the expected range for $\mathrm{He}, \mathrm{He}_{2}, \mathrm{He}_{3}$, and $\mathrm{Ne}$ interacting with a transition metal surface [4,18]. According to Refs. [4,18], $C_{3}$ for $\mathrm{He}$ is $\sim 3.2-4.3\left(10^{-50} \mathrm{~J} \mathrm{~m}^{3}\right)$ since this is the expected range for the interaction with a transition metal surface. For $\mathrm{He}_{2}$, one expects $l$ to be the same as He but $C_{3}$ to be two times larger. The same proportionality for $C_{3}$ is expected for $\mathrm{He}_{3}$. This has been our guide to fit the interaction potentials for the three $\mathrm{He}$ clusters. For the Ne case, one expects larger values for $l$ and $C_{3}$ but our only guide has been the fitting to the corresponding experimental results. The different potential parameters for

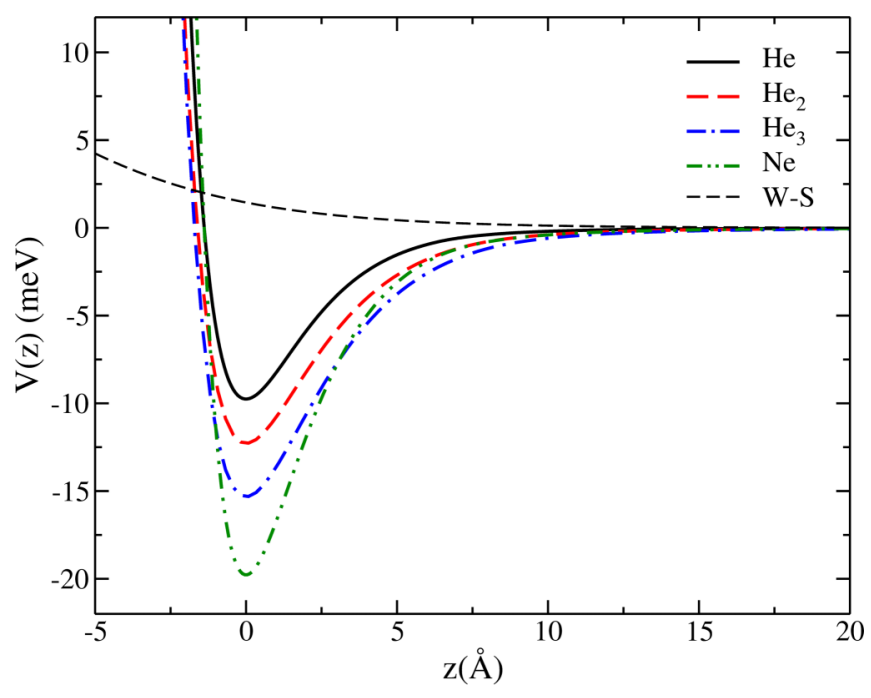

FIG. 1. Vertical interaction potentials $V(z)$ between the incident particles and the grating. The black dashed curve is the WS absorbing potential used. This potential is added as an imaginary part for all diffraction channels with slightly modified parameters (see text).

the four systems used here seem to be plausibly close to values obtained from $a b$ initio arguments or calculations [4,18]. The vertical interaction potentials $V(z)$ for the four systems and the WS potential are plotted in Fig. 1. As expected, the well depth of the Morse potential increases with the mass of the incident particle.

The WS potential (the dashed black curve in Fig. 1) is "turned on" in the region of the classical turning point of the interaction potential, in particular, at the initial value of the grid $z_{i}$. Quantum reflection is not observed when the absorbing potential is placed far to the right of the potential well, where the vdW-Casimir tail of the interaction potential is prevalent. In other words, the inner part of the interaction potential has a profound effect on the reflection probability, showing that this region cannot be omitted from the $z$-grid integration. This is again confirmed by the different values of the Morse potential parameters for the different incident particles. All of the diffraction channels are modified by adding the imaginary WS potential. The couplings among them do not involve any imaginary part. The two parameters $A$ and $\alpha$ needed to define the WS potential for every diffraction channel are chosen after several runs of the CC code. In a first run, those parameters are varied by including only three diffraction channels: the specular channel $(n=0)$ and the two diffraction channels labeled by $n= \pm 1$, with the demand being that the specular reflection probability is similar to that of the one-channel calculation. In a second run, the next two diffraction channels $n= \pm 2$ are added by using the same criterion. This general numerical procedure is relatively straightforward to implement because the corresponding parameters of the remaining channels are much less sensitive to the total quantum reflection. The resulting parameters are listed in Table II.

In Fig. 2, the quantum reflection probabilities are plotted versus the perpendicular incident wave vector $k_{\text {perp }}$ (in $\mathrm{nm}^{-1}$ ) for (i) $\mathrm{He}$ and $\mathrm{He}_{2}$ with incident energy $T_{0}=20$ and 
TABLE II. Parameters $A$ and $\alpha$ of the WS potential used for the incident particles $\mathrm{He}, \mathrm{He}_{2}, \mathrm{He}_{3}$, and Ne. The initial grid points are $z_{i}=-10,-20,-21,-12 \AA$, respectively. The parameters for each system are written as $\left(A_{n}, \alpha_{n}\right)$ where the subindices 0 and 1 are used for the specular channel $(n=0)$ and the first-order diffraction channels $n= \pm 1$, respectively, and the subindex 2 is used for the remaining diffraction channels. $A$ values are in atomic units. Numbers in parentheses mean powers of 10 ; for example $7.0(-4) \equiv 7.0 \times 10^{-4}$.

\begin{tabular}{lcccr}
\hline \hline Parameters & $\mathrm{He}$ & $\mathrm{He}_{2}$ & $\mathrm{He}_{3}$ & $\mathrm{Ne}$ \\
\hline$\left(A_{0}, \alpha_{0}\right)$ & $(7.0(-4), 0.5)$ & $(2.0(-6), 0.1)$ & $(2.0(-3), 0.3)$ & $(2.0(-2), 0.9)$ \\
$\left(A_{1}, \alpha_{1}\right)$ & $(9.0(-5), 0.1)$ & $(9.0(-5), 0.1)$ & $(2.0(-1), 0.5)$ & $(2.0(-2), 1.5)$ \\
$\left(A_{2}, \alpha_{2}\right)$ & $(7.0(-3), 0.3)$ & $(4.0(-2), 0.3)$ & $(2.0(-4), 0.1)$ & $(2.0(-2), 0.12)$ \\
\hline \hline
\end{tabular}

$T_{0}=15 \mathrm{~K}$, respectively, with $\theta_{i}=3.4,5.2,7.6,9.1,12.1$, $15.1,18.9 \mathrm{mrad}$, (ii) $\mathrm{He}_{3}$ with incident energy $T_{0}=8.7 \mathrm{~K}$ and $\theta_{i}=0.8,1.1,1.2,1.4,1.6 \mathrm{mrad}$, and (iii) $\mathrm{Ne}$ with incident energy $T_{0}=40 \mathrm{~K}$ and $\theta_{i}=0.5,0.6,0.7,0.8,1.1,1.3 \mathrm{mrad}$. Circles, triangles and squares labels represent the experimental values of Ref. [18] and color curves correspond to the multichannel calculation. The overall agreement between theory and experiment is fairly good. The results corresponding to the He dimer are a prediction since no experimental information exists in the literature for the regular grating used in our computations. The absorbing potential has negligible effects on the theoretical quantum reflection probabilities.

As clearly seen in this figure, the reflection probabilities at a given value of $k_{\text {perp }}$ are much smaller for massive incident particles. They are quite similar for $\mathrm{He}$ and $\mathrm{He}_{2}$ on the one hand and $\mathrm{He}_{3}$ and $\mathrm{Ne}$, on the other hand. Experimental confirmation would be highly desirable to validate the theoretical behavior of the He dimer. As previously reported [11], the full interaction region (inner and external regions) contributes coherently and equally to the reflection probabilities. In particular, at higher perpendicular wave vectors, incident particles explore deeper and deeper regions of the potential well, the attractive part is no longer described by the vdW-Casimir tail.

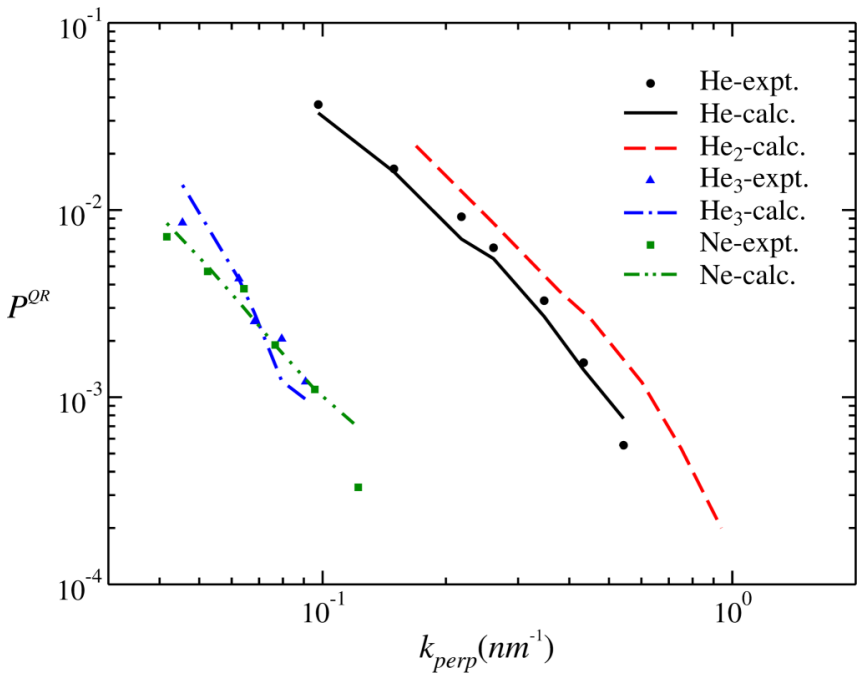

FIG. 2. Theoretical (multichannel calculations) quantum reflection probabilities for $\mathrm{He}\left(T_{0}=20 \mathrm{~K}\right), \mathrm{He}_{2}\left(T_{0}=15 \mathrm{~K}\right), \mathrm{He}_{3}\left(T_{0}=\right.$ $8.7 \mathrm{~K})$, and $\mathrm{Ne}\left(T_{0}=40 \mathrm{~K}\right)$ vs the perpendicular incident wave vector $k_{\text {perp }}\left(\right.$ in $\mathrm{nm}^{-1}$ ). The results for the He dimer are not compared with experiment since they have been measured only for scattering from a blazed grating rather than the regular grating used here. The experimental points have been provided by the authors of Refs. [4,7].
This part is considered in our model by means of a Morse potential which is much more appropriate.

The expected linear dependence of the quantum reflection probability on the incident perpendicular wave vector is revealed only at very small incident perpendicular wave vectors as seen in Fig. 3. The theoretical (multichannel calculations) quantum reflection probabilities are plotted versus $k_{\text {perp }}$ (in $\mathrm{nm}^{-1}$ ) for $\mathrm{He}, \mathrm{He}_{2}, \mathrm{He}_{3}$, and $\mathrm{Ne}$ but keeping the same incident de Broglie wavelength fixed at $\lambda=0.179 \mathrm{~nm}$. The source temperature $T_{0}$ used for each incident particle is given in parentheses. Here too, the slopes in the linear regime depend on the incident mass, the lighter the particle the smaller is the slope. As previously mentioned, the characteristic length $b$ governing the slope of the linear regime is determined in a region where the potential differs appreciably from zero. The linear behavior is a direct result of boundary conditions and continuity of the wave function and its derivative and is thus "universal." However, the magnitude of the slope is system specific and depends globally on the interaction potential. The linear regime is no longer observed when increasing the perpendicular wave vector, showing a new functional dependence with the wave vector $[12,14]$

When the same incident wave vector is used for the four diffractive systems, it is also quite illustrative to demonstrate

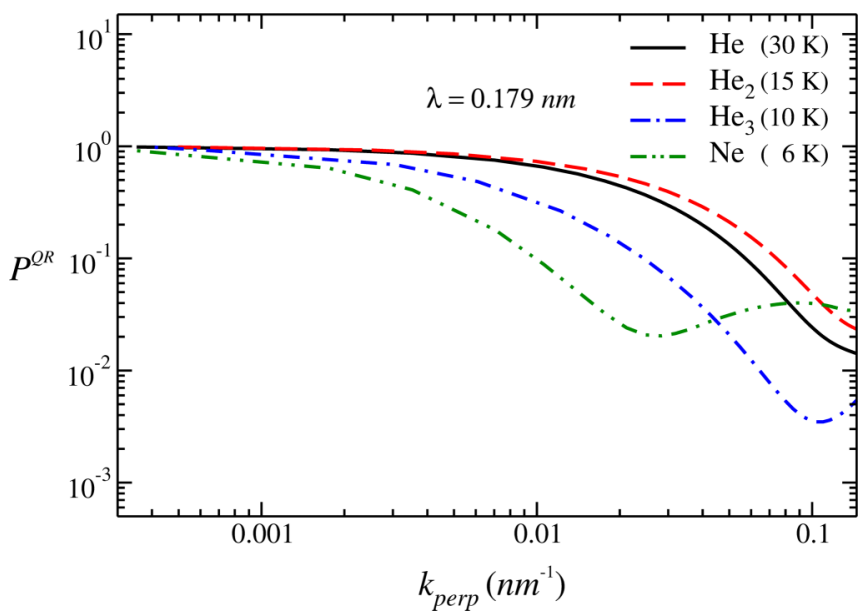

FIG. 3. Quantum reflection probabilities vs $k_{\text {perp }}$ (in $\mathrm{nm}^{-1}$ ) for $\mathrm{He}, \mathrm{Ne}, \mathrm{He}_{2}$, and $\mathrm{He}_{3}$ at the same de Broglie wavelength, $0.179 \mathrm{~nm}$. The source temperature $T_{0}$ used for each system is given in parentheses. Note that the reflection probabilities only display a universal linear behavior at very small $k_{\text {perp }}$. At higher values of $k_{\text {perp }}$, the behavior is quite similar for the heavier masses $\left(\mathrm{He}_{3}, \mathrm{Ne}\right)$ and lighter particles $\left(\mathrm{He}, \mathrm{He}_{2}\right)$. 


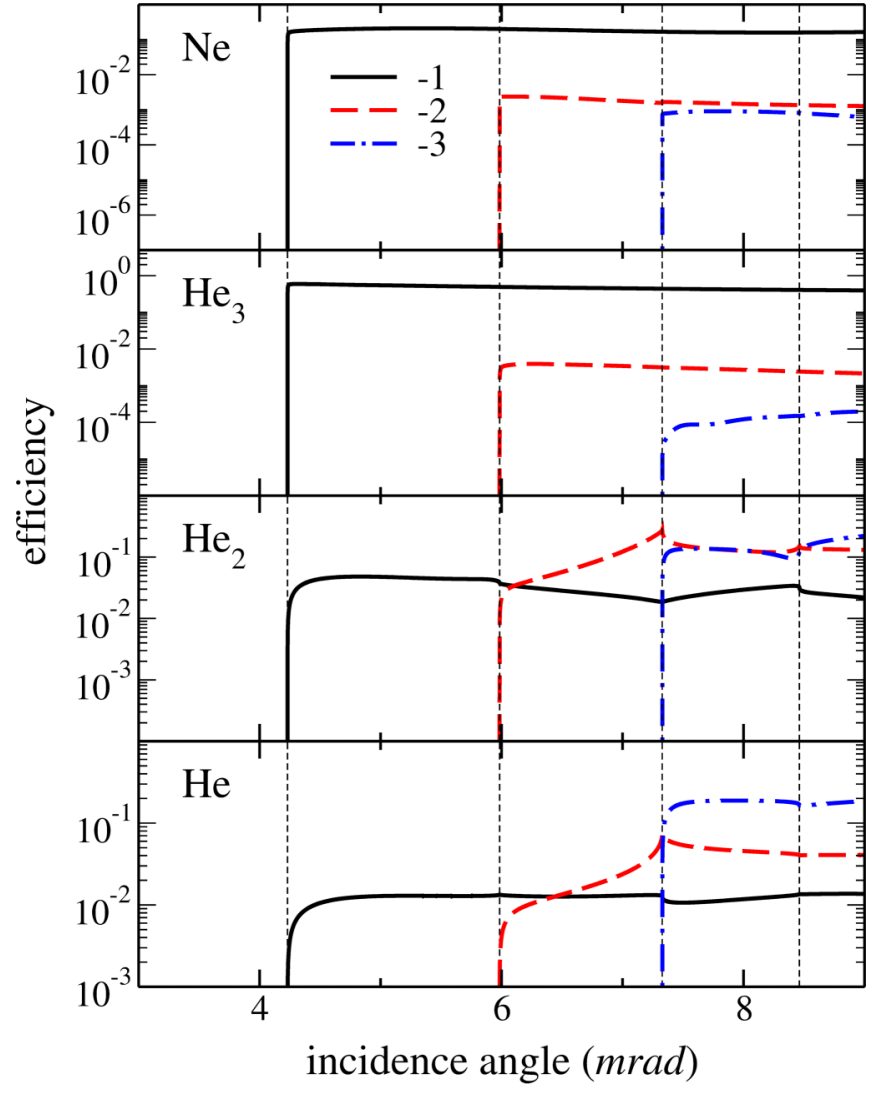

FIG. 4. Diffraction efficiencies vs the incident angle (in mrad) for $\mathrm{He}, \mathrm{Ne}, \mathrm{He}_{2}$, and $\mathrm{He}_{3}$ at the same de Broglie wavelength of $\lambda=$ $0.179 \mathrm{~nm}$. The Rayleigh angles are plotted as dashed vertical lines to indicate the opening or closing of a given diffraction channel.

the universal dependence of the Rayleigh angles on the incident angle [4]. In Fig. 4, diffraction efficiencies are plotted as a function of the incident angle (in mrad) for the different incoming particles as well as the Rayleigh angles (in dashed vertical lines) showing when the diffraction channels $-1,-2$, -3 just become open. This occurs for any integer value of $n$ at which the kinematic relation [Eq. (11)] exactly vanishes. As may be inferred from the kinematic relation, the Rayleigh angles are then functions of the de Broglie wavelength of the incident particle and the lattice length only. Therefore, when scattering different particles on the same grating, one should expect that the threshold angles are only a function of the de Broglie wavelength. This is clearly seen in Fig. 4. At the Rayleigh angles, abrupt changes of the diffraction intensities are observed for the four systems (four panels) due to the redistribution of the intensities among the open channels. This is also the typical behavior displayed in atom-surface scattering [10].

It has also been observed in Ref. [4] that when plotting the efficiency of the $n=-1$ diffraction peak as a function of the incident angle on a logarithmic scale that it is only a function of the de Broglie wavelength of the incident particle. This was observed using a blazed grating. In Fig. 5 we plot the efficiencies we obtained for the diffraction channel $n=-1$ on a linear scale as a function of the incidence angle, keeping the incident de Broglie wavelength fixed and normalizing the plots

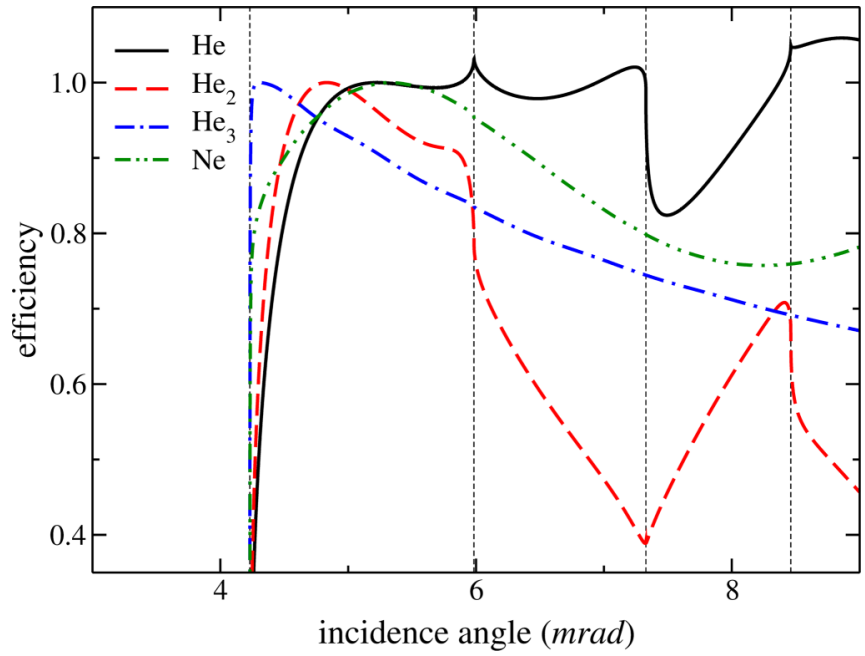

FIG. 5. Diffraction efficiencies of the open $n=-1$ diffraction channel vs the incident angle (in mrad) for $\mathrm{He}, \mathrm{Ne}, \mathrm{He}_{2}$, and $\mathrm{He}_{3}$ at the same de Broglie wavelength of $\lambda=0.179 \mathrm{~nm}$. The Rayleigh angles are plotted as dashed vertical lines to indicate the opening or closing of a given diffraction channel.

for the different particles to unity at their maximum. It is clear that our theoretical results do not show any universal behavior in this context. The dependence on the angle of incidence changes when using different particles and is determined by the overall potential and identity of the incident particle.

\section{CONCLUDING REMARKS}

We have demonstrated, using the close-coupling formulation for scattering and employing absorbing boundary conditions, that it is possible to account theoretically for the quantum reflection probabilities found experimentally when scattering $\mathrm{He}, \mathrm{He}_{3}$, and $\mathrm{Ne}$ from a regular grating. We have also used the same to predict the quantum reflection probabilities expected for the $\mathrm{He}_{2}$ dimer. The same computations were then used to verify the universal dependence of the so-called emerging resonances, or Rayleigh angles and their dependence on the incident de Broglie wavelength alone, as also observed experimentally in Ref. [4]. Finally, we have shown that one should not expect a universal dependence of the diffraction efficiency on the incident angle and wave number.

We believe the physics underlying these results is quite clear. Quantum reflection is a coherent, nonlocal phenomenon where all the regions (inner and outer) of the interaction potentials must be considered on an equal footing. In particular, the boundary condition which imposes the vanishing of the wave function for sufficiently small distances from the grating implies that the quantum reflection has very little to do with the so-called badlands region of the long-range attractive potential. The de Broglie wavelength of the incident particle is much longer than the spatial extent of the badlands region. It is this large wavelength which is critical for understanding the quantum reflection phenomenon. The wave function covers all regions of the potential so that all of them affect the final reflection probability. The Morse potential used for describing 
the short-range interaction is a convenient way to cover the whole interaction region. Quantum reflection does not occur at tens or hundreds of nanometers away from the grating. Although the maximal density of the outgoing wave function is found very far from the surface, as in many other quantum phenomena, the wave function at any position is affected by any other position. Just as in the two-slit problem, the wave function far away from any of the slits is affected by the wave function at the slits; so here, the wave function at its maximum, which is far from the surface, is affected by the wave function close to the surface.

The implication of this is that, as observed in our computations, the magnitude of the linear slope of the reflection probability as a function of the incident perpendicular wavelength is system and potential specific. Similarly, the diffraction efficiencies are found to be system specific. Only the emerging resonances are universal since they are a kinematic effect, which signals the opening up of new diffraction channels. The main difference between our computations and the experimental results presented in Ref. [4] is that we use a regular grating rather than a blazed grating. Only quantitative differences in the final results are expected when using a blazed surface. In this context we note that the theoretical results presented here for the $\mathrm{He}_{2}$ dimer are a prediction which could be validated using new experiments with a regular grating rather than a blazed one. There is no reason a priori to prevent using more sophisticated interaction potentials in this formalism as used in Ref. [26].

\section{ACKNOWLEDGMENTS}

We thank W. Schöllkopf and B. S. Zhao for providing us with their experimental results. This work is supported by the Programa Nacional de Ciencias Básicas de Cuba PNCB: P223LH001-108 (G.R.L. and J.R.S.), by a grant with Ref. FIS2017-83473-C2-1-P from the Ministerio de Ciencia, Innovación y Universidad (Spain) (S.M.A.), and by grants of the Israeli Science Foundation, the Minerva Foundation, Munich and the German Israel Foundation for Basic Research (E.P.).
[1] M. Berry, I. Marzoli, and W. Schleich, Phys. World 14, 39 (2001).

[2] A. S. Sanz and S. Miret-Artés, J. Chem. Phys. 126, 234106 (2007).

[3] S. Gerlich, L. Hackermüller, K. Hornberger, A. Stibor, H. Ulbricht, M. Gring, F. Goldfarb, T. Savas, M. Müri, M. Mayor, and M. Arndt, Nat. Phys. 3, 711 (2007).

[4] B. S. Zhao, W. Zhang, and W. Schöllkopf, Sci. Adv. 2, e1500901 (2016).

[5] J. E. Lennard-Jones and A. F. Devonshire, Proc. R. Soc. London A 156, 6 (1936).

[6] W. Kohn, Surf. Sci. Lett. 1, 129 (1994).

[7] B. S. Zhao, G. Meijer, and W. Schöllkopf, Science 331, 892 (2011).

[8] H. Friedrich and A. Jurisch, Phys. Rev. Lett. 92, 103202 (2004).

[9] T. A. Pasquini, Y. Shin, C. Sanner, M. Saba, A. Schirotzek, D. E. Pritchard, and W. Ketterle, Phys. Rev. Lett. 93, 223201 (2004).

[10] R. Guantes, F. Borondo, J. Margalef-Roig, S. Miret-Artés, and J. R. Manson, Surf. Sci. 375, L379 (1997).

[11] S. Miret-Artés and E. Pollak, J. Phys. Chem. Lett. 8, 1009 (2017).

[12] J. Petersen, E. Pollak, and S. Miret-Artés, Phys. Rev. A 97, 042102 (2018).
[13] H. Friedrich, G. Jacoby, and C. G. Meister, Phys. Rev. A 65, 032902 (2002).

[14] P. Senn, Am. J. Phys. 56, 916 (1988).

[15] H. Friedrich and J. Trost, Phys. Rep. 397, 359 (2004).

[16] A. R. Barnea, B. A. Stickler, O. Cheshnovsky, K. Hornberger, and U. Even, Phys. Rev. A 95, 043639 (2017).

[17] A. S. Sanz and S. Miret-Artés, Phys. Rep. 451, 37 (2017).

[18] B. S. Zhao, S. A. Schulz, S. A. Meek, G. Meijer, and W. Schöllkopf, Phys. Rev. A 78, 010902(R) (2008).

[19] O. Kornilov and J. P. Toennies, Europhys. News 38, 22 (2007).

[20] L. W. Bruch, W. Schöllkopf, and J. P. Toennies, J. Chem. Phys. 117, 1544 (2002).

[21] B. A. Stickler, U. Even, and K. Hornberger, Phys. Rev. A 91, 013614 (2015).

[22] B. S. Zhao, G. Meijer, and W. Schöllkopf, Phys. Rev. Lett. 104, 240404 (2010).

[23] G. Armand and J. R. Manson, Surf. Sci. 169, 216 (1986).

[24] T. Seideman and W. H. Miller, J. Chem. Phys. 98, 4412 (1992).

[25] J. G. Muga, J. P. Palao, B. Navarro, and I. L. Egusquiza, Phys. Rep. 395, 357 (2004).

[26] A. M. Contreras-Reyes, R. Guérout, P. A. M. Neto, D. A. R. Dalvit, A. Lambrecht, and S. Reynaud, Phys. Rev. A 82, 052517 (2010). 